\title{
Giant Infective Endocarditis of Native Aortic Valve with Secondary Mitral Kissing Vegetation
}

\author{
Andrea Cardona ${ }^{1 *}$, Giuseppe Vittorio De Socio², Ketty Savino, Stefano Pasquino ${ }^{3}$ and Giuseppe Ambrosio \\ ${ }^{1}$ Department of Cardiology and Cardiovascular Pathophysiology, University of Perugia, Italy \\ ${ }^{2}$ Department of Infectious Diseases, Santa Maria della Misericordia Hospital, Perugia Italy \\ ${ }^{3}$ Deaprtment of Cardio surgery, Santa Maria della Misericordia Hospital, Perugia Italy
}

\begin{abstract}
Secondary involvement of the mitral valve is well documented in primary aortic valve endocarditis. An important causative mechanism, involving both left-sided valves, is 'mitral kissing vegetation'. This results from large aortic vegetations prolapsing into the left ventricular outflow tract and "kissing" with the ventricular surface of the anterior mitral leaflet thus causing secondary infection.
\end{abstract}

Keywords: Endocarditis; Echocardiography; Cardiac valve disease; Vegetation; Aortic valve; Mitral valve

\section{Case Report}

A previously healthy 50 -year-old male was admitted to the hospital because of acute congestive heart failure and fever. He reported a long history of illness with fever round to $38.2^{\circ} \mathrm{C}$, began two months before. Hypertension and mild dyslipidemia were the only cardiovascular risk factors to note. Physical examination revealed tachycardia, a grade $3 / 6$ diastolic aortic murmur, $2 / 6$ systolic mitral murmur, pulmonary congestion and peripheral hypoperfusion. Laboratory exams showed leukocytosis, anemia, microhematuria, elevated erythrocyte sedimentation rate and C-reactive protein. Three sets of blood culture yielded Streptoccus mutans. The isolate was susceptible to penicillin with minimal inhibitory concentration of $0.016 \mathrm{mg} / \mathrm{L}$. Transthoracic echocardiography was then performed showing multiple areas of increased echogenicity both on the aortic and mitral valve. Biventricular function was preserved. The patient was treated with standard doses of intravenous antibiotic regimen of ceftriaxone and gentamycin for diagnosis of endocarditis. On transesophageal echocardiography mid esophageal long- and short-axis views showed multiple giant vegetations $(>15 \mathrm{~mm})$ involving all aortic leaflets, causing global disarrangement of their anatomy and severe aortic regurgitation (white arrows, Figure 1a and 1b). Aortic leaflets presented rupture at the base of implant with important diastolic flail in the left ventricular
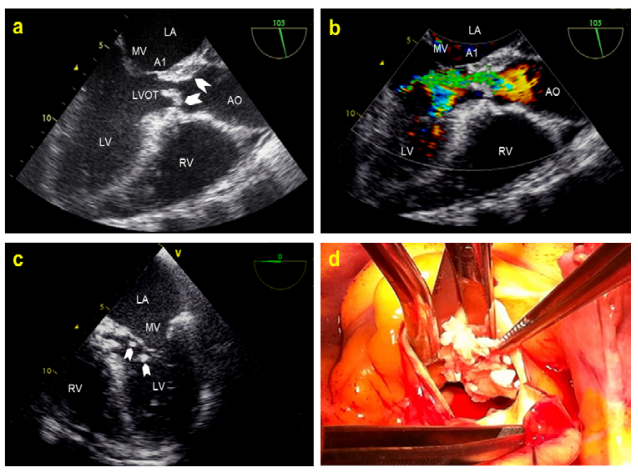

Figure 1: a: 2-D gray scale of mid esophageal long axis view $\left(105^{\circ}\right)$. b: $2-D$ color-Doppler of mid esophageal long axis view $\left(105^{\circ}\right)$. c: $2-D$ gray scale of mid esophageal five chamber view $\left(0^{\circ}\right)$. d: Intraoperative image of giant aortic endocarditis.

Abbreviations: LV: Left Ventricle; RV: Right Ventricle; AO: Aorta; MV: Mitral Valve; LA: Left Atrium; LVOT: Left Ventricle Outflow Tract; A1: Anterolateral Portion of the Anterior Mitral Leaflet. outflow tract, and "kissing" of the ventricular surface of the anterior mitral leaflet (figure, panels a-b-c). The 5-chamber view (Figure 1c) showed multiple, highly mobile vegetations involving anterior and posterior mitral leaflets (white arrows, Figure 1c), spreading along subvalvular apparatus. Despite adequate antimicrobial therapy, an emergency surgical intervention was performed due to rapidly deteriorating hemodynamics [1]. Intraoperatively, massive aortic valve incompetence due to rupture of left and non-coronary leaflets at the base was evident. Aortic cusps were thickened; irregular and friable (Figure 1d). Papillary muscles appeared pale and ischemic. Mitral and aortic valves were replaced by bio prosthesis. Postoperative course was uneventful.

\section{Discussion}

Secondary infection of the mitral valve, though uncommon, is a possible finding in primary aortic valve endocarditis. An important causative mechanism, involving both left-sided valves, is the "mitral kissing vegetation" [2]. This results from large aortic vegetation prolapsing, during diastole, into the left ventricular outflow tract, "kissing" the ventricular surface of the anterior mitral leaflet, and thus causing secondary infection. This is not a common phenomenon; in the study of Piper and colleagues, only 19 out of 192 patients with primary aortic valve endocarditis, studied by serial transesophageal echocardiography, showed secondary involvement of mitral valve [2]. Although relatively rare, "mitral kissing vegetation" can lead to an increased risk of complications and worse outcome. In fact patients with aortic valve endocarditis plus "mitral kissing vegetation" show higher prevalence of embolic events, renal failure and have larger aortic valve vegetations $(>6 \mathrm{~mm})$ as compared to patients with aortic valve endocarditis alone [2-4]. Of note, not only primary aortic endocarditis may be the causative mechanism for secondary mitral valve involvement. In fact, although extremely rare, left ventricle

*Corresponding author: Andrea Cardona, Department Cardiology and Cardiovascular Pathophysiology, University of Perugia, Ospedale Santa Maria della Misericordia, piazzale Menghini, 1-06129 Perugia, Italy, Tel: +39 075 5851; E-mail: andreacardona20@yahoo.it

Received August 30, 2014; Accepted October 26, 2014; Published October 28 2014

Citation: Cardona A, De Socio GV, Savino K, Pasquino S, Ambrosio G (2014) Giant Infective Endocarditis of Native Aortic Valve with Secondary Mitral Kissing Vegetation. J Clin Case Rep 4: 425. doi:10.4172/2165-7920.1000425

Copyright: ( $) 2014$ Cardona A, et al. This is an open-access article distributed under the terms of the Creative Commons Attribution License, which permits unrestricted use, distribution, and reproduction in any medium, provided the original author and source are credited. 
Citation: Cardona A, De Socio GV, Savino K, Pasquino S, Ambrosio G (2014) Giant Infective Endocarditis of Native Aortic Valve with Secondary Mitral Kissing Vegetation. J Clin Case Rep 4: 425. doi:10.4172/2165-7920.1000425

outflow tract endocarditis may represent the initial site of infection with possibility to spread by contiguity to both mitral and aortic valves [4].

\section{References}

1. Habib G, Hoen B, Tornos P (2009) ESC Committee for Practice Guidelines. Guidelines on the prevention, diagnosis, and treatment of infective. The Task Force on the Prevention, Diagnosis, and Treatment of Infective Endocarditis of the European Society of Cardiology (ESC). Endorsed by the European Society of Clinical Microbiology and Infectious Diseases (ESCMID) and the International Society of Chemotherapy (ISC) for Infection and Cancer. Eur Heart J. 30: 2369-2413.
2. Piper C, Hetzer R, Körfer R, Bergemann R, Horstkotte D (2002) The importance of secondary mitral valve involvement in primary aortic valve endocarditis; the mitral kissing vegetation. Eur Heart J 23: 79-86.

3. Becker AE (1974) Cardiac complications of infectious endocarditis of the aortic valve. Eur J Cardiol 1: 467-472.

4. lacovelli F, Scicchitano P, Zanna D, Marangelli V, Favale S (2012) Left ventricle outflow tract vegetation, embolism and troponin rise: an infective endocarditis case report. Intern Emerg Med 7 Suppl 2: S145-147. 